13

\title{
A way to increase the bit rate in ionospheric radio links
}

\author{
CLENCY PERRINE $\left({ }^{1}\right)$, YVON M. ERHEL $\left({ }^{2}\right)$, DOMINIQUE LEMUR $\left({ }^{1}\right), \operatorname{LOUIS~BERTEL~}\left({ }^{1}\right)$ \\ and ALAIN BOURDILLON $\left(^{1}\right)$ \\ ( $\left.{ }^{1}\right)$ Institut d'Electronique et Télécommunications de Rennes, Université de Rennes 1, France \\ ( $^{2}$ ) Institut d'Electronique et Télécommunications de Rennes, Centre de Recherches \\ des Ecoles de Coëtquidan, Guer, France
}

The aim of this paper is to present a high data rate transmission system through the ionospheric channel in the HF-band (3-30 MHz). The applications expected in this study are image transmitting and real-time videoconferencing. Very high rates are required compared to the standard modems. Therefore, an array processing is performed with a set of antennas whose spatial response differs from one another arranged in a circular array or in a collocated sensor. Synchronization (Zero Crossing Detector) and source separation (LMS algorithm) resort to classical well-tested techniques involving training sequences. Experimental results are presented for both antenna configurations. These techniques improve data rate, reaching $20 \mathrm{kbits} / \mathrm{s}$ within the $6 \mathrm{kHz}$ bandwidth (QAM 64) without coding or interleaving.

\subsection{INTRODUCTION}

HF-waves (3-30 MHz), when propagated through the ionosphere, can achieve very long distance transmissions with a minimal infrastructure compared to satellite links, for example. This multipath multimode channel strongly degrades transmissions (mainly fading and frequency selectivity). For this reason, the data rate of standard scalar HF modems does not exceed $4.8 \mathrm{kbps}$ in $3 \mathrm{kHz}$ bandwidth.

The originality of this study is to use a heterogeneous array to improve the HF transmission. Indeed, it has been shown that such a device could achieve the direction finding regarding the incoming polarization as a decorrelation factor and, consequently, that the diversity of the spatial responses could, to some extent, replace space diversity (Erhel et al., 1998).

The array processing is performed on a set of four active collocated antennas or a circular array and the corresponding coherent receiving channels. The spatio-temporal equalization resorts to the LMS (Least Mean Square) algorithm as the synchronisation is based on a «Zero Crossing Detector».

In order to introduce the heterogeneous array and to bring its advantages, it is necessary to start with a short description of ionospheric propagation and the effects on transmission. Then it is shown that a heterogeneous array can improve the HF transmission and the real transmission system is described. Finally, experimental results are provided to illustrate the theory by practical examples. 


\subsection{Propagation of HF-WAVES in the iONOSPhere}

\subsubsection{Multipath and multimode}

Two main phenomena must be considered in ionospheric propagation. The first is that waves can follow several distinct paths by reflecting themselves against different layers of the ionosphere. Then the transmitted signal gives birth to as many replicas that are affected in different ways by the channel: their differential time delays can be large, and their Doppler shifts and angles of arrival can be spaced out too. The second phenomenon appears because of the Earth's magnetic field that makes the medium anisotropic. Within each path, the wave splits into two complementary propagation modes called $O$ (ordinary) and $X$ (extraordinary). Both are very close and therefore have similar time delays, Doppler shifts and DOA's (Direction Of Arrival). On the other hand, their polarization properties are very different. In the plane orthogonal to the direction of propagation, their electromagnetic fields describe orthogonal ellipses clockwise and counterclockwise (Budden, 1985). Multipath and multimode propagation are illustrated in fig. 13.1.

\subsubsection{Effects on transmission}

All the transmitted signal replicas created by multipath and multimode propagation interfere, degrading transmission quality. Again, two cases can be distinguished that do not have the same consequences, although they are of the same nature. If two signals are delayed by an interval smaller than $1 / B$ (where $B$ is their bandwidth), they interfere in a way that the resultant signal fades, making the Signal to Noise Ratio (SNR) vary in time (an example is given in fig. 13.2). In the opposite case, the SNR remains constant but frequency selectivity occurs.

Fading is due to multimode propagation. Only the $O$ - and $X$-modes of the same path can be close enough to cause it. It is the most important problem for transmitting data since the signal is susceptible to completely disappear for a while. Concerning frequency selectivity, it is caused by modes coming from distinct paths. When several modes are present, both phenomena may be observed. The time-frequency analysis of the signal displayed in fig. 13.2 is plotted in fig. 13.3. It represents the mapping of its power in the time-frequency plane, and is obtained directly through the observation of the broadband modulated signal (Bisiaux, 2001). Two sets of interference patterns are clearly visible, generating both fading and frequency selectivity. They denote the presence of at least 3 modes, among which 2 come from the same path (the line spectrum that appears every $4 \mathrm{~s}$ is not a computation aberration, it is due to a periodic pattern inserted into the transmitted signal).

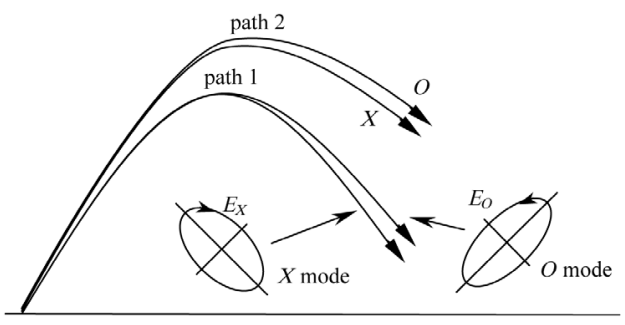

Fig. 13.1. Multipath and multimode propagation in the ionosphere. 

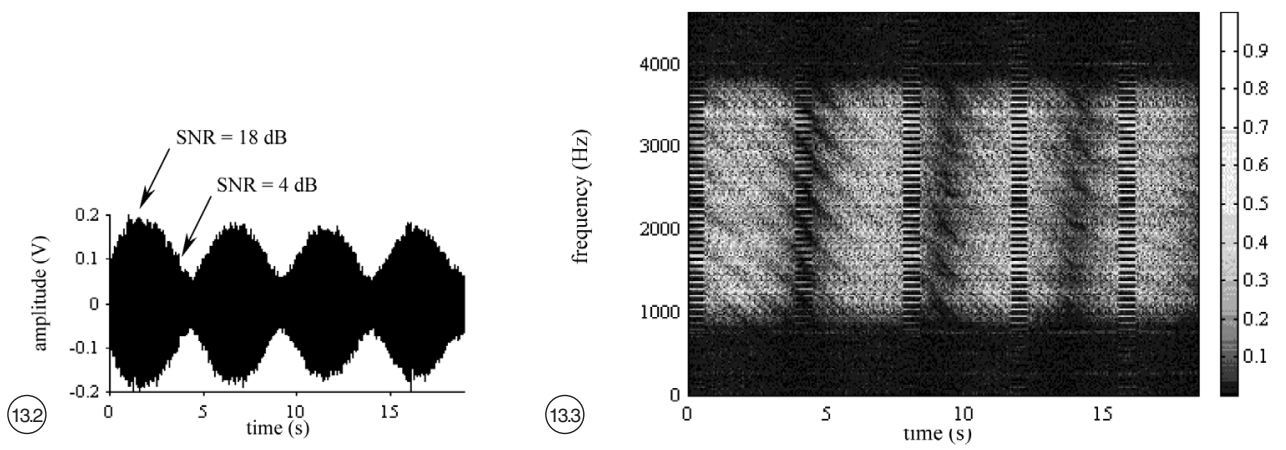

Fig. 13.2. Example of a signal fading (QAM-16, $3 \mathrm{kHz}$ bandwidth, $F \sim 8 \mathrm{MHz}$ ).

Fig. 13.3. Time-frequency analysis of an HF signal at Intermediate Frequency IF $=2.25 \mathrm{kHz}$.
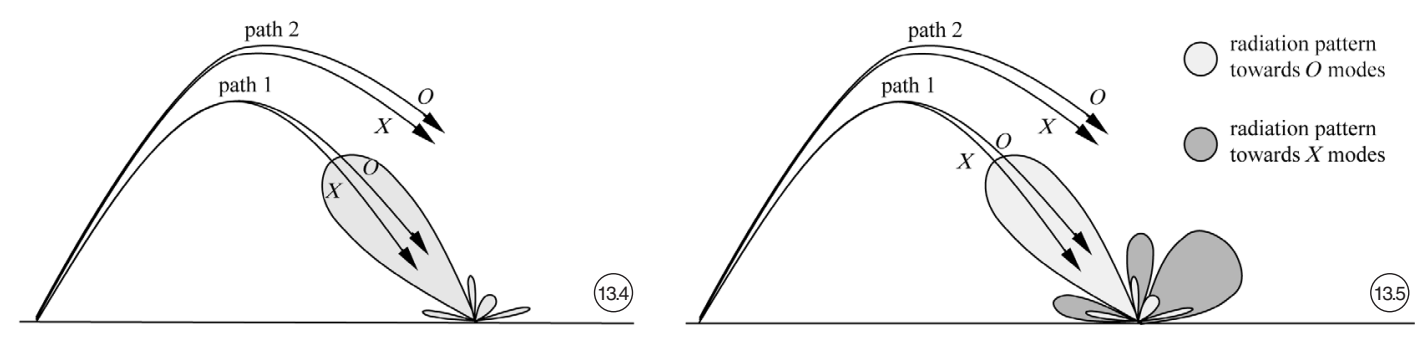

Fig. 13.4. Source separation with a classical array.

Fig. 13.5. Source separation with a heterogeneous array.

\subsubsection{Improvement of $\mathrm{HF}$ transmissions resorting to arrays of receiving antennas}

\subsubsection{Classical arrays}

Most of the time, arrays are made of identical elements set distant from one another (they are homogeneous arrays). Space diversity allows them to steer their main lobe toward the DOA of a desired signal, or to create notches in the direction of undesired ones. However, such arrays would not be a very good solution for HF communications applications. The reason for that is illustrated in fig. 13.4: they would be efficient to combat multipath effects, but would not be able to separate the complementary modes of a single path because they usually leave the ionosphere at very close angles. The spatially filtered signal would then still be affected by fading. Furthermore, given that the wavelength ranges from 10 to $100 \mathrm{~m}$, such arrays would spread over a large area, making them rather impractical. For these reasons, homogeneous arrays are rarely used for transmission purposes in the HF-band. Yet it is possible to suppress fading by separating the $O$ - and $X$-modes, but it requires including some kind of polarization treatment in the whole process, which is not possible with homogeneous arrays. 


\subsubsection{Heterogeneous arrays}

With antenna diversity, it is possible to join the ability of an array to alter its radiation pattern on the one hand, and polarization filtering on the other hand. This leads to the realization of a heterogeneous array, that is to say, made of antennas with various shapes or orientations, as opposed to classic homogeneous arrays. Its application to HF transmissions can be illustrated in a simplified way by the drawing in fig. 13.5: the radiation pattern of the array is not the same for all the waves because it depends on their polarization. Since $O$ - and $X$-modes coming out of the ionosphere have different polarization properties, two different radiation patterns must be considered instead of one, as is usually the case. One regards $O$-modes, and is completely unrelated to the other one that concerns $X$-modes. For this reason it is theoretically possible to separate two complementary modes, even if they come from the same direction, and still being able to create notches in the two radiation patterns. Such a principle is ideal for combating both multipath and multimode propagation.

As a consequence, the steering vector of a heterogeneous array is expressed as

$$
a_{h O / X}=\left[\begin{array}{l}
F_{10 / X} \cdot e^{j \varphi 2(\theta)} \\
F_{2 o / X} \cdot e^{j \varphi 2(\theta)} \\
\cdots \\
F_{N C O I X} \cdot e^{j \varphi 2(\theta)}
\end{array}\right]
$$

where the $\varphi_{\kappa}(\theta)$ are the geometrical phases of a classical array and $F_{n_{O X}}$ is the spatial response of antenna $n$ for the two expected polarizations.

\subsubsection{Expressions of the spatial correlation}

For an array of isotropic sensors the spatial correlation of two incident sources with DOA $\theta_{1}$ and $\theta_{2}$ is expressed as

$$
\rho=\frac{\boldsymbol{a}\left(\theta_{1}\right)^{H} \boldsymbol{a}\left(\theta_{2}\right)}{\left\|\boldsymbol{a}\left(\theta_{1}\right)\right\| \cdot\left\|\boldsymbol{a}\left(\theta_{2}\right)\right\|}=\frac{\sum_{n=1}^{N C} e^{j}\left[\varphi_{n}\left(\theta_{2}\right)-\varphi_{n}\left(\theta_{1}\right)\right]}{N C}
$$

Using a heterogeneous array with the same geometry, the expression of the spatial correlation becomes in a general case (whatever is the incident polarization)

$$
\rho=\frac{\boldsymbol{a}\left(\theta_{1}\right)^{H} \boldsymbol{a}_{h}\left(\theta_{2}\right)}{\left\|\boldsymbol{a}_{h}\left(\theta_{1}\right)\right\| \cdot\left\|\boldsymbol{a}_{h}\left(\theta_{2}\right)\right\|}=\frac{\left[\boldsymbol{F}\left(\theta_{1}\right) \otimes \boldsymbol{a}\left(\theta_{1}\right)\right]^{H} \boldsymbol{F}\left(\theta_{2}\right) \otimes \boldsymbol{a}\left(\theta_{2}\right)}{\left\|\boldsymbol{F}\left(\theta_{1}\right) \otimes \boldsymbol{a}\left(\theta_{1}\right)\right\| \cdot\left\|\boldsymbol{F}\left(\theta_{2}\right) \otimes \boldsymbol{a}\left(\theta_{2}\right)\right\|}
$$

where $\boldsymbol{F}(\theta)$ is the vector of the spatial responses for the DOA $\theta$.

This quantity can be written under the following form:

$$
\rho_{h}=\frac{\sum_{n=1}^{N C} \boldsymbol{F}_{n}^{*}\left(\boldsymbol{\theta}_{1}\right) \cdot \boldsymbol{F}_{n}\left(\boldsymbol{\theta}_{2}\right) e^{j}\left[\varphi_{n}\left(\theta_{2}\right)-\varphi_{n}\left(\theta_{1}\right)\right]}{\sqrt{\sum_{n=1}^{N C}\left|\boldsymbol{F}_{n}\left(\boldsymbol{\theta}_{1}\right)\right|^{2}} \cdot \sqrt{\sum_{n=1}^{N C}\left|\boldsymbol{F}_{n}\left(\boldsymbol{\theta}_{2}\right)\right|^{2}}}
$$

The modulus of the spatial correlation is modified for this type of array: $\left|\rho_{h}\right| \neq|\rho|$. 


\subsubsection{Improvement due to the heterogeneous array}

A comparison of the two quantities $\left|\rho_{h}\right|$ and $|\rho|$ does not provide a general result: the heterogeneity of the array does not systematically decrease the spatial correlation. However, it can be mentioned that current situations of reception are characterized by very close angles of arrival though the spatial responses are significantly different for the DOA $\theta_{1}$ and $\theta_{2}$ : it is the case with diversely polarized electromagnetic waves impinging from the same direction.

The geometrical phases are nearly equal in these conditions $\left(\varphi_{n}\left(\theta_{1}\right) \cong \varphi_{n}\left(\theta_{2}\right)\right)$ and the modulus of the spatial correlation for the homogeneous array is close to $1:|\rho| \approx 1$.

On the other hand, considering the correlation for the heterogeneous array, the modulus $\left|\rho_{h}\right|$ approaches the value

$$
\left|\rho_{h}\right| \cong \frac{\left|\sum_{n=1}^{N C} \boldsymbol{F}_{n}^{*}\left(\theta_{1}\right) \cdot \boldsymbol{F}_{n}\left(\theta_{2}\right)\right|}{\sqrt{\sum_{n=1}^{N C}\left|\boldsymbol{F}_{n}\left(\theta_{1}\right)\right|^{2}} \cdot \sqrt{\sum_{n=1}^{N C}\left|\boldsymbol{F}_{n}\left(\theta_{2}\right)\right|^{2}}} .
$$

This ratio is less or equal to one, according to the Schwartz inequality: the spatial correlation decreases in this case.

\subsubsection{Numerical simulation}

In the following simulation, HF radio waves are assumed to be received after propagation through the ionosphere. It involves two uniform circular arrays made up with 8 loop antennas (figs. 13.6 and 13.7). This geometry has a practical advantage since the accuracy of the angular estimation is not dependant on the actual azimuth of arrival in this case. The first array is made up with 8 identical sensors (homogeneous array) and its spatial correlation is $\rho$. In the second one, the antennas are submitted to a rotation of $30^{\circ}$ around a vertical axis every two positions within the array, so
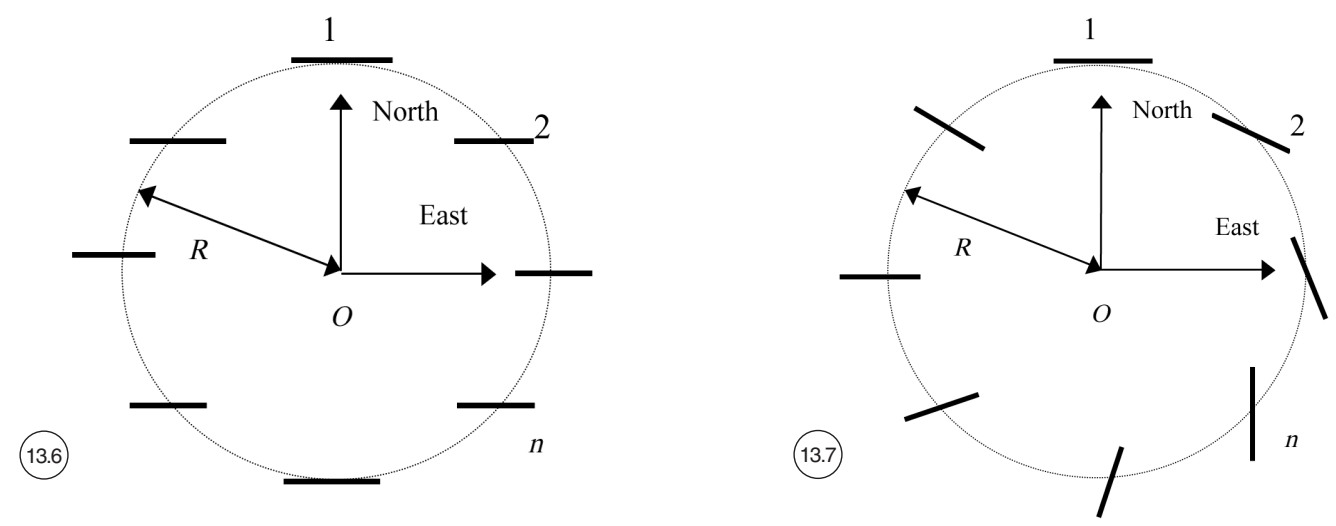

Fig. 13.6. Homogeneous circular array.

Fig. 13.7. Heterogeneous circular array. 


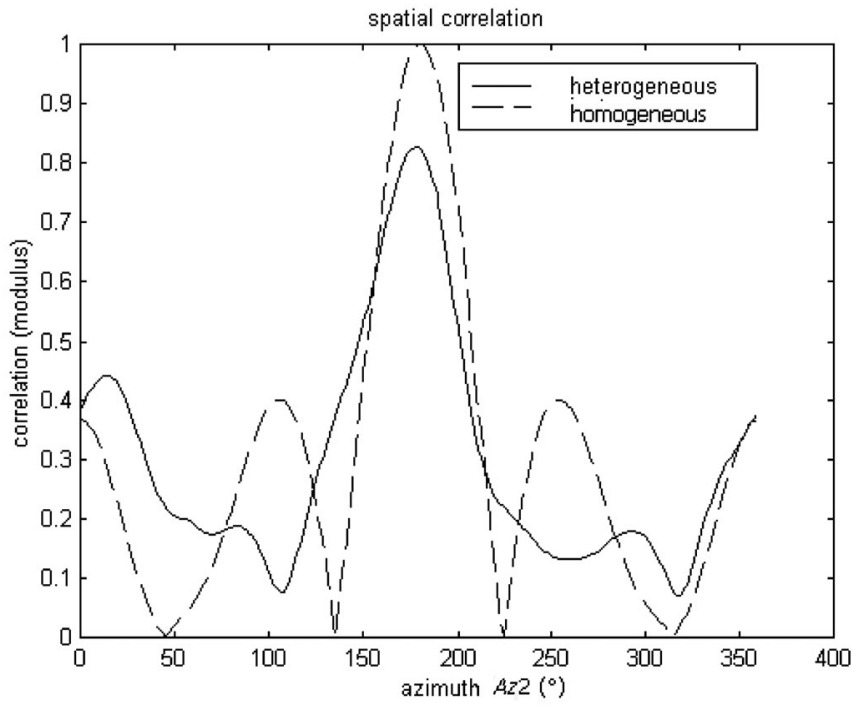

Fig. 13.8. Comparison of the spatial correlation of signals.

that it becomes heterogeneous with a spatial correlation $\rho_{h}$. This angular value realizes a compromise between a significant diversity of the spatial responses and the existence of redundancy if the rotation exceeds $180^{\circ}$. The spatial responses of the antennas are computed according to the model described in Section 13.2. The numerical parameters are: radius $R=0.5 \lambda, \lambda$ denoting the wavelength, and carrier frequency fo $=15 \mathrm{MHz}$.

Two incident waves are impinging with a common elevation and orthogonal polarizations. The first signal has a fixed azimuth of arrival $\left(A z 1=180^{\circ}\right)$, and the second azimuth $A z 2$ varies in the range of $0^{\circ}$ to $360^{\circ}$. The simulation compares the spatial correlation of this couple of incident waves (considered as a function of the variable azimuth $A z 2$ ) for the homogeneous and the heterogeneous array. Whatever the method of direction finding, the less is the modulus of the spatial correlation, the easier is the separation of the signals. The critical case corresponds to a modulus close or equal to one for which the separation fails.

Figure 13.8 plots the spatial correlations for both arrays (modulus of $\rho$ and $\rho_{h}$ ) as function of the variable azimuth $A z 2$. The benefit provided by the heterogeneous array appears in an interval of $A z 2$ containing the value of $A z 1=180^{\circ}$. In that area, the modulus $\left|\rho_{h}\right|$ is significantly smaller than $|\rho|$.

In particular, in the critical case of two superimposed incident waves $\left(A z 2=A z 1=180^{\circ}\right)$, the modulus $\left|\rho_{h}\right|$ is equal to 0.82 and $|\rho|$ to 1 : the heterogeneous array can separate the incident waves (with orthogonal polarizations) while the homogeneous array cannot. $\left|\rho_{h}\right|$ is not less than $|\rho|$ whatever the azimuth, but when it exceeds this reference value, the corresponding values are significantly smaller than 1 , so that the DOA estimation works correctly.

\subsection{THE TRANSMISSION SYSTEM}

The link used for the experiments has been established between Poitiers (latitude: $46^{\circ} 35^{\prime} \mathrm{N}$, longitude: $0^{\circ} 25^{\prime} \mathrm{E}$ ) and Monterfil (latitude: $48^{\circ} 03^{\prime} \mathrm{N}$, longitude: $2^{\circ} 00^{\prime} \mathrm{O}$ ) near Rennes (both in 
France). It is about $300 \mathrm{~km}$ long. Figure 13.9 gives a schematic view of the link and of the devices employed. This is a short link for an HF transmission that is normally used to cover several thousands of kilometers.

\subsubsection{The transmitting system}

The transmitting system is located in Poitiers. In order to provide a good flexibility, it was decided that the whole digital signal processing would be done with a computer: digital modulation and filtering, possibly interleaving and coding, etc. This way it is possible to control very easily the type of modulation used (PSK, QAM, OFDM, ...), the sampling frequency and the signal's bandwidth.

The system is composed of a personal computer, a complex modulator, an HF power amplifier connected to a delta antenna. The complex modulator's architecture is shown in fig. 13.9. Its role is simply to convert digital data sent in real-time by the PC into analog signals, through the means of DACs (Digital to Analog Converters), and then to transpose them into the HF-band. The operation is complex in that both real and imaginary parts of the complex baseband signal ( $I$ and $Q$ channels respectively) are sent to the modulator, which also generates a complex carrier with a DDS (Direct Digital Synthesiser).

Let $x(t)=a(t)+j b(t)$ be the complex baseband signal. The synthesized signal can be expressed as

$$
s(t)=a(t) \cos (\omega t)-b(t) \sin (\omega t) .
$$

which represent the real part of the equivalent complex signal (called analytical signal)

$$
s(t)=\Re\left\{x(t) e^{j \omega t}\right\} .
$$

The carrier frequency is programmable through the $\mathrm{PC}$, from 0 to $13 \mathrm{MHz}$ by $0.01 \mathrm{~Hz}$ steps. The phase shift between channels I and Q of the DDS is adjustable too, which can be used to compensate for phase errors due to electronic components. The sampling frequency can be set between $180 \mathrm{kHz}$ and $1 \mathrm{MHz}$ with $20 \mathrm{~ns}$ steps in period. It should be set high enough in order to make the rejection of the mirror spectrum easy, while enabling the emission of a wide-band signal. However, it remains limited by the throughput of the PC's bus, which must send data in real-time. The DACs are followed by low-pass filters with a $100 \mathrm{kHz}$ cut-off frequency. Thus the maximum bandwidth of the signal is $200 \mathrm{kHz}$.

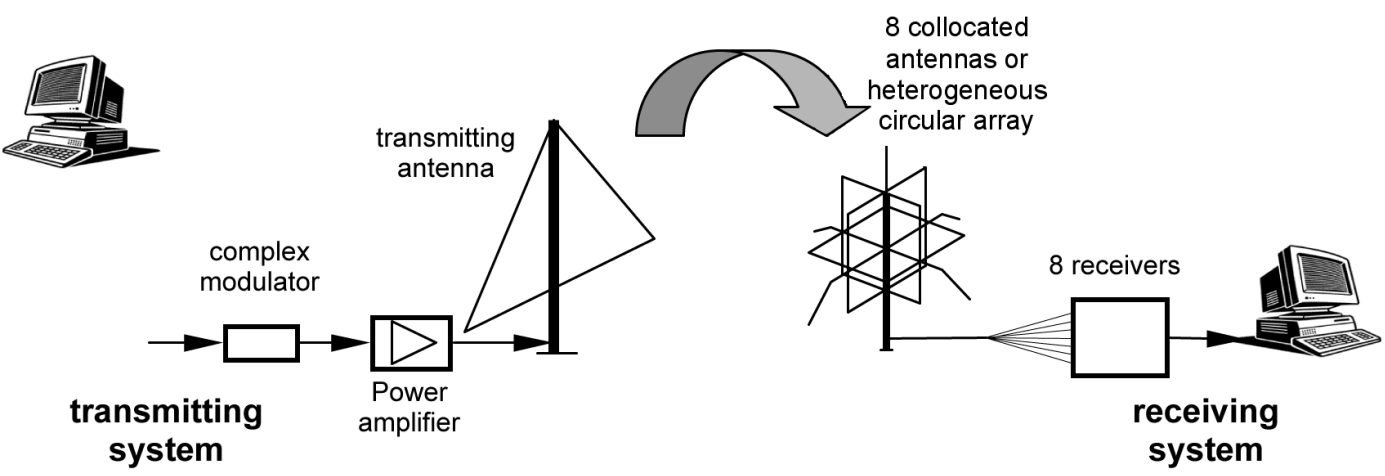

Fig. 13.9. Schematic of the Poitiers-Monterfil link. 


\subsubsection{The receiving system}

The system used as a receiver is located in Monterfil. It was at first designed as a direction finding system and sounder, and then adapted to the current application. Therefore it is not as flexible as the transmitter.

The array is a set of 8 collocated active antennas, as described by Marie et al. (2000) or a heterogeneous circular array, as depicted in fig. 13.7. The collocated array is followed by 8 identical receivers which transpose the incoming HF signals onto a $30 \mathrm{kHz}$ intermediate frequency. Then they are filtered using a quartz filter with either with a $3 \mathrm{kHz}$ or a $12 \mathrm{kHz}$ bandwidth. The sampling is performed at a programmable rate and the data is stored on the PC disk.

The most restrictive features of the receiver are its bandwidth (12 kHz maximum), which might become a concern in future experiments, and the access time of the PC's hard drive. Indeed, a few seconds of data result in files of several megabytes which must be saved in real-time and too long acquisitions often make the acquisition board overflow.

\subsubsection{Spatio-temporal equalization}

As NS sources are incident on the array, the signal at the output of one antenna has the following form:

$$
x_{i}(t)=\sum_{k=1}^{N S} \boldsymbol{F}_{i}\left(\theta_{k}\right) s_{k}(t)+n_{i}(t)
$$

A source separation method tends to estimate a set of weighting factors wik so that the filtered signal

$$
\hat{S}_{k}=\sum_{1}^{N C} w_{i k} x_{i}(t)
$$

is the closest estimate of $s_{k}(t)$.

In the application, the classical Least Mean Square (LMS) algorithm is processed. The goal is to minimize the power of the error between the estimated signal $\hat{s}_{k}(t)$ and the reference signal $s_{k}$ (Widrow and McColl, 1976) resorting to a descent along the gradient. It is assumed that a training sequence is transmitted to make the estimation of the weighting factors converge. Then, the effective data transmission begins and their real-time computation involves the estimated received data instead of the training data.

\subsubsection{Synopsis of the signal processing performed at reception}

In the complete receiving system which has been developed, the array processing is only one among a set of functions including:

- matched filtering (to the transmitted wave form);

- phase and frequency synchronisation;

- time synchronisation (optimal placing of the sampling times);

- interpolation.

Figure 13.10 summarizes all these operations. The input signals $x_{i}(t)$ are the received signals transposed in base band. Their phases are corrected by the phase synchronisation loop. The synchronisation techniques are based on an approach of the maximum of likelihood running with the spatially filtered signal. Each symbol is over sampled with a ratio of 10: $T / T_{e}=10$ if $T$ denotes the symbol duration and $T_{e}$ the sampling period. 


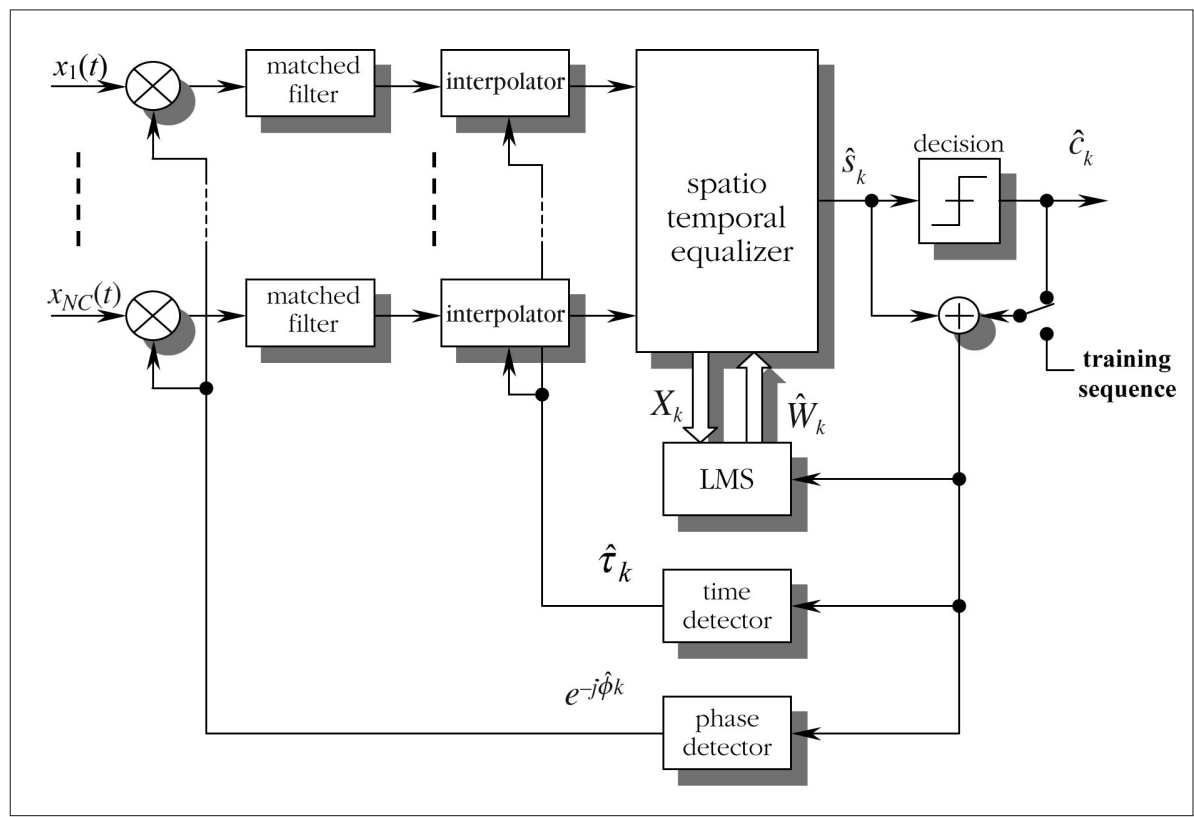

Fig. 13.10. Synopsis of the receiver.

A good temporal resolution in the placing of the sample times needs an interpolation: a parabolic interpolator with 4 coefficients has been used. The loop yields an estimate $\hat{\tau}_{k}$ of the time delay at the $k t h$ iteration; its recursive expression integrates an error signal generated by a «zero crossing detector»

$$
\hat{\tau}_{k+1}=\hat{\tau}_{k}+\gamma e_{k}
$$

with

$$
e_{k}=\Re\left\{\left(\hat{c}_{k-1}^{*}-\hat{c}_{k}^{*}\right) \hat{s}\left(k T-T / 2+\hat{\tau}_{k-1}\right)\right\} .
$$

The quantities with a star denote the conjugates of the estimated complex data and the sample of the filtered signal is picked up with a rate twice faster than the symbol rate (Mengali and D'Andrea, 1997).

\subsection{Results}

\subsubsection{Characteristics of the signals received using the 4 collocated antennas}

The results presented in this section are based on an example that reflects the behavior of the system in the majority of cases. Only 4 antennas were used out of the 8 available ones because the other 4 proved to be unnecessary: they did not help to improve the transmission performances due a low level of signals for the tested link and, moreover, they induced a larger computational time. The 4 elements of the array, as pictured in fig. 13.11, are:

- a vertical square loop contained in the East-West plane; 

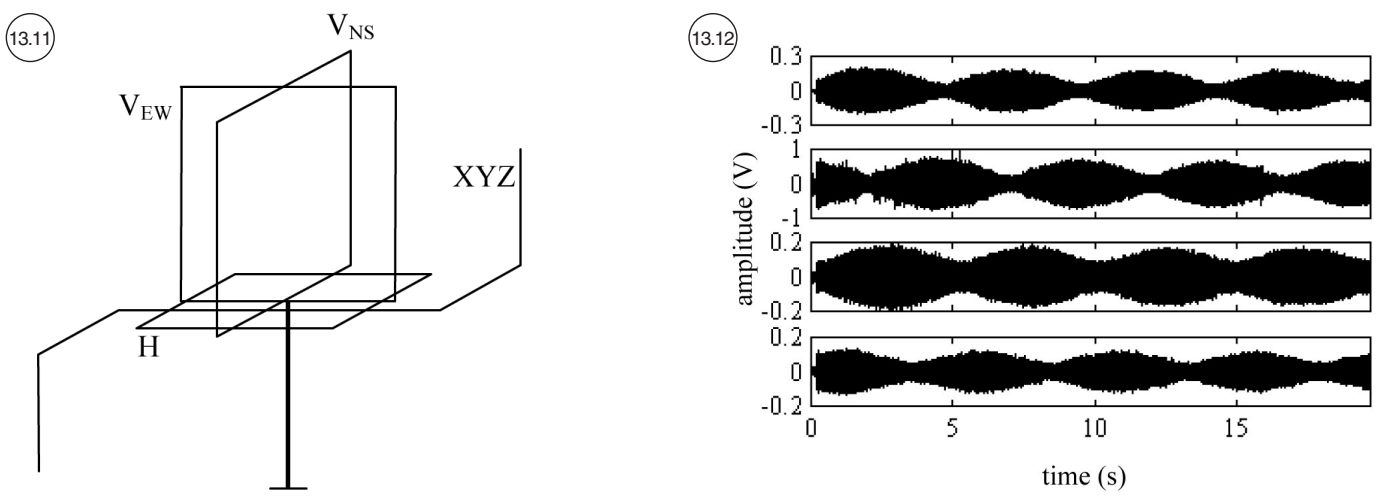

Fig. 13.11. Schematic of the collocated array actually used (4 antennas).

Fig. 13.12. Signals received by the array $\left(\mathrm{V}_{\mathrm{EW}}, \mathrm{V}_{\mathrm{NS}}, \mathrm{H}, \mathrm{XYZ}\right)$.
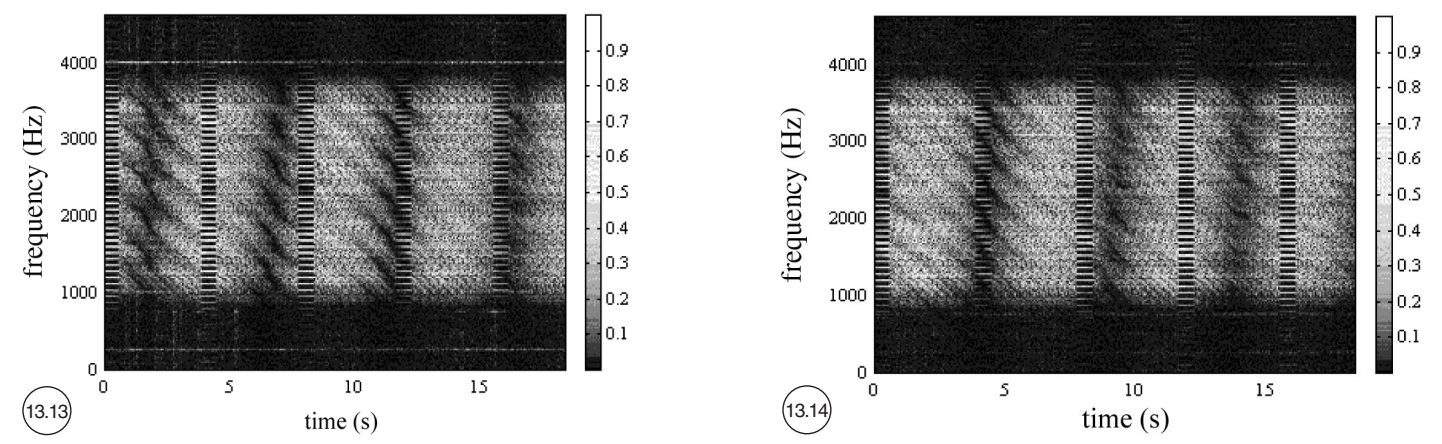

Fig. 13.13. Time-frequency analysis of the second channel at IF $\left(\mathrm{V}_{\mathrm{NS}}\right)$.

Fig. 13.14. Time-frequency analysis of the first channel at $\mathrm{IF}\left(\mathrm{V}_{\mathrm{EW}}\right)$.

- a vertical square loop contained in the North-South plane;

- a horizontal square loop;

- an XYZ dipole.

The transmitted signal has the following properties:

- 16-QAM modulation with rolloff factor of 0.2 ;

- $3 \mathrm{kHz}$ bandwidth;

- 10 kbps (2500 baud);

- carrier frequency of $8 \mathrm{MHz}$.

The received signals are plotted in fig. 13.12. They are all affected by fading, which denotes that propagation is mainly provided by 2 complementary modes of a principal path. In such a case, these modes come from very close directions, however the array's antenna diversity is enough to introduce a significant difference between the 4 observations. This remark is reinforced by the time-frequency representations of the first two signals (figs. 13.13 and 13.14). Two interference patterns appear (fig. 13.3); in addition to fading (the most visible one), some frequency selectivity shows that a second 
path also exists. But for these two interference patterns, the signal fades at different instants and frequencies from one antenna to the other, what underlines the decorrelation provided by the diversity.

\subsubsection{The heterogeneous array: a way to improve HF transmissions}

The adaptive method based on the LMS algorithm has been applied successively to 2, 3 and 4 antennas in order to evaluate the influence of the array's size.

- 2 antennas: the propagation conditions are too constraining during the first seconds of the transmission and the algorithm is unable to keep track. However, it starts to converge again after $6 \mathrm{~s}$ and then the BER remains below $10^{-2}$ independently of the fading (fig. 13.15). The average BER in this case is $2 \times 10^{-2}$, not much higher than in the best case with a simple polarization filter. Obviously, two antennas are not enough to realize a reliable filter.

- 3 antennas: this time the algorithm converges immediately (fig. 13.16) and the BER always remains below $3 \times 10^{-2}$ with a mean value of $3.7 \times 10^{-3}$.

- 4 antennas: since the previous case led to very good results, the fourth antenna does not bring as much change as the third one (fig. 13.17), but still the BER is improved with an average of $2.8 \times 10^{-3}$.

The SNR obtained in the latter case (after adaptive filtering) is reproduced in fig. 13.18, along with the raw SNR received on the first antenna (the same pattern can be observed on the 3 others with
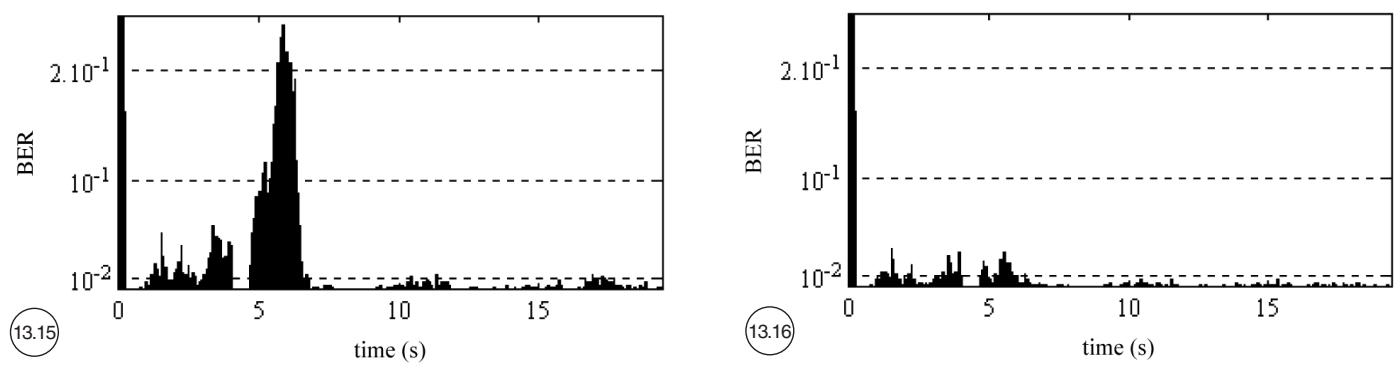

Fig. 13.15. BER obtained using 2 antennas.

Fig. 13.16. BER obtained using 3 antennas.
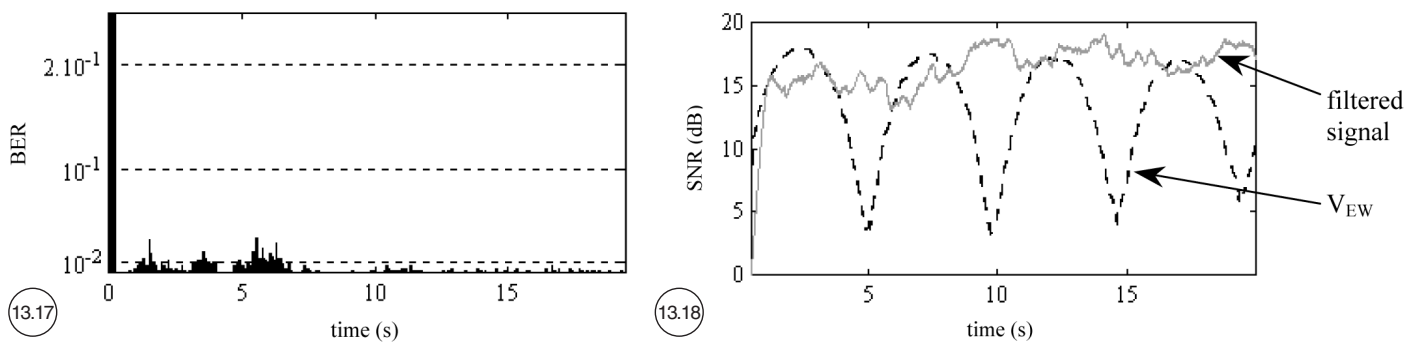

Fig. 13.17. BER obtained using 4 antennas.

Fig. 13.18. Comparison of $S N R$ on antenna $V_{E W}$ with $S N R$ after filtering. 
fading occurring at different times). This figure shows the advantage of antenna diversity: whatever the method employed to demodulate the signal, the quality of the transmission can only be improved when the SNR remains almost constant and is not subject to deep fading.

\subsubsection{Experimental results with the array of 4 collocated antennas}

The following experiments are obtained with 4 collocated antennas as shown in fig. 13.11. Several transmissions have been made at the end of year 2000 with carrier frequencies between 6 and 9 $\mathrm{MHz}$ (between Poitiers and Monterfil). Acquisitions were done over $20 \mathrm{~s}$. No coding, interleaving or soft decision was employed.

\subsubsection{Use of a $3 \mathrm{kHz}$ bandwidth}

The waveform is a QAM-16 or QAM-64 modulation. Its envelope has a roll-off factor equal to 0.2 and the symbol duration is equal to $0.4 \mathrm{~ms}$ (2500 bauds).

\subsection{First example: QAM-16}

The results presented here have been obtained for a typical case where deep fading appears, due to interferences between two close complementary modes. Figure 13.19 shows the set of 4 signals at the array output. Their minima and maxima do not occur at the same time, which proves that their association can bring diversity other than space diversity.

The complex samples which are collected at the output of the spatial filtering (before the hard decision) are plotted in fig. 13.20. However, the most energetic symbols are not exactly centered at the corners of the map: that indicates that the power stage of the transmitter is not linear for corresponding signals. The corresponding Bit Error Rate (BER) is plotted in fig. 13.21. The mean value is $2.7 \times 10^{-3}$ and the data rate is $9700 \mathrm{bps}$.
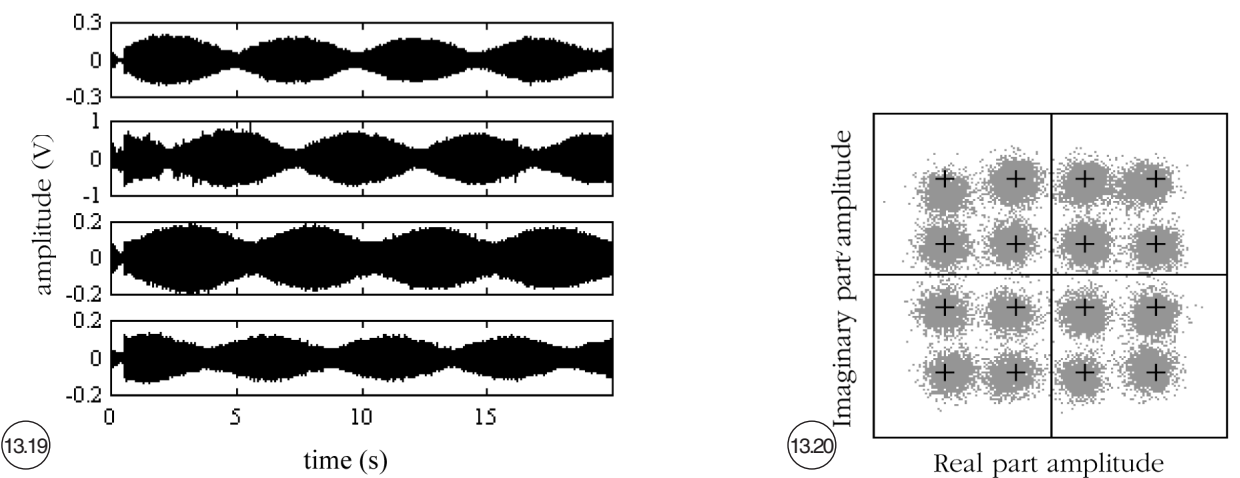

Fig. 13.19. Four channel acquisition.

Fig. 13.20. Constellation at the output of the spatial filter. 

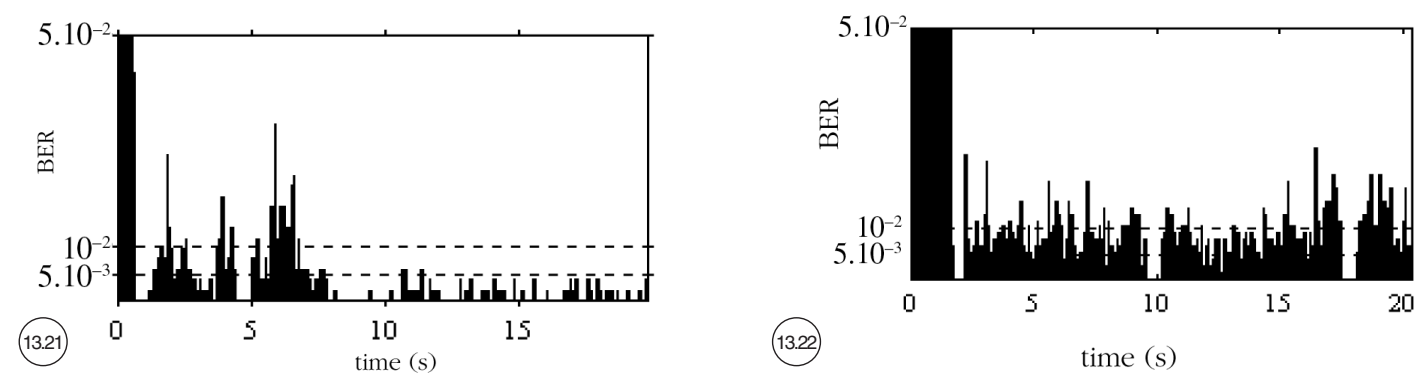

Fig. 13.21. Variations of BER.

Fig. 13.22. Temporal variations of BER.
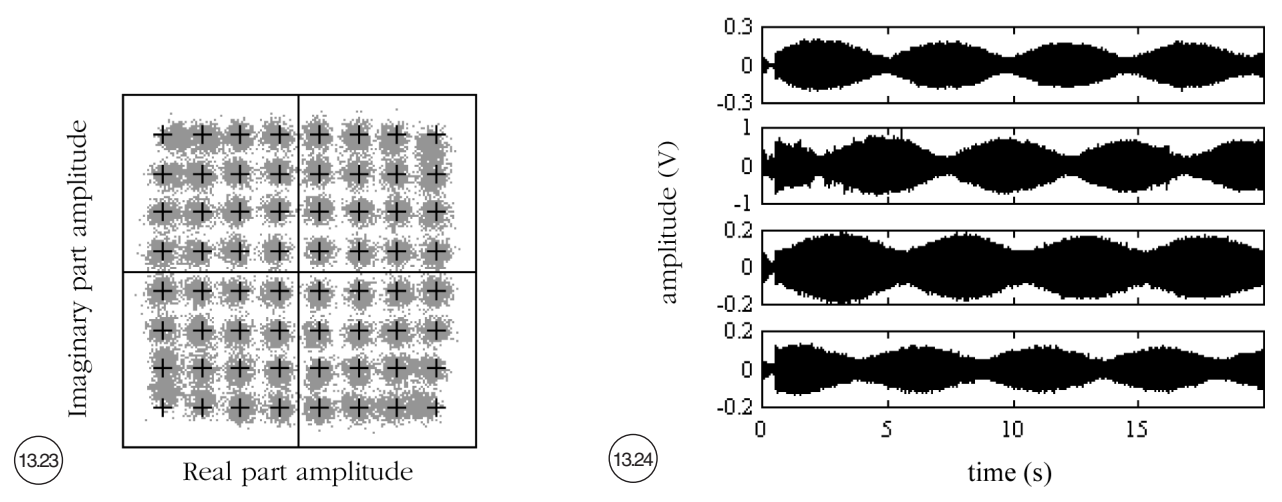

Fig. 13.23. Constellation with a QAM-64.

Fig. 13.24. Signals received on the 4 collocated antennas.

\subsection{Second example: QAM-64}

In the following experiment, the waveform was a QAM-64 modulation and the bit transfer rate equal to $15 \mathrm{kbps}$. The average signal to noise ratio (before the spatial filtering) equals $20 \mathrm{~dB}$. The corresponding bit error rate is plotted in fig. 13.22 and the complex samples collected at the output of the spatial filtering are plotted in fig. 13.23. In this case the mean BER value is $7.2 \times 10^{-3}$.

\subsubsection{Use of a $6 \mathrm{kHz}$ bandwidth}

The waveform is a QAM-16 modulation. Its envelope has a roll-off factor equal to 0.2 and the symbol duration is equal to $0.2 \mathrm{~ms}$ (5000 bauds) involving a bandwidth of $6 \mathrm{kHz}$. If the channel parameters are still the same, the SNR is decreased by $3 \mathrm{~dB}$. Figure 13.24 shows the set of 4 signals delivered at the array output. The mean SNR is $18 \mathrm{~dB}$. The variations of the BER is plotted in fig. 13.25 and the mean value is $2.3 \times 10^{-4}$. The data rate is $19.4 \mathrm{kbps}$ and the corresponding constellation is plotted in fig. 13.26. 

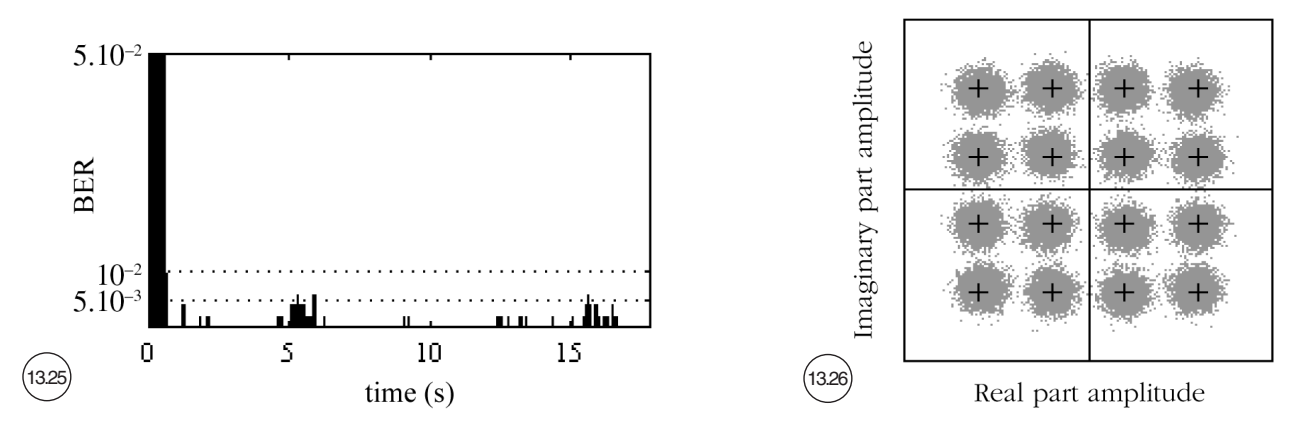

Fig. 13.25. Temporal variations of the BER.

Fig. 13.26. Constellation at the output of the spatial filter.

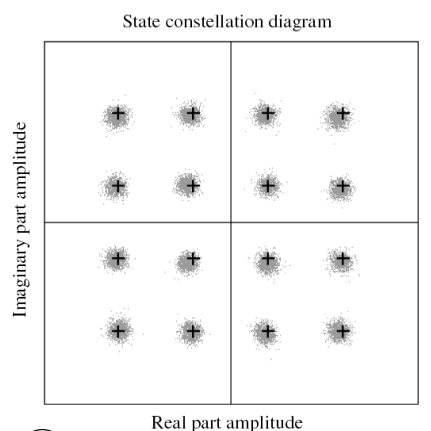

(13.27)

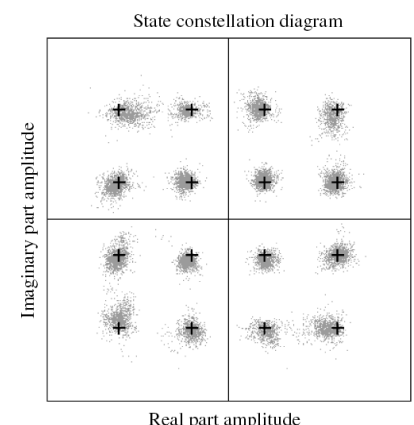

(13.28)

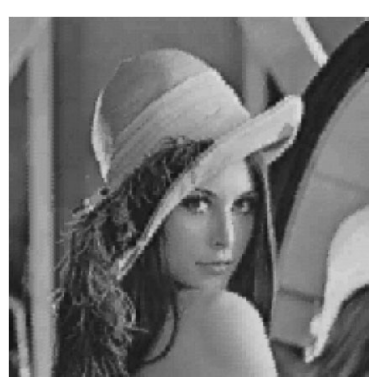

(13.29)

Fig. 13.27. Constellation at the output of the spatial filter.

Fig. 13.28. State constellation diagram (6.7 MHz).

Fig. 13.29. Original transmitted image.

\subsubsection{Experimental results: heterogeneous circular array of 4 loop antennas}

The following experiments involved a heterogeneous circular array of 4 loop antennas and the transmitted data are images. The goal is to design a still image transmission system with a good reception quality in presence of transmissions errors (Souhard et al., 2003). That work was developed at the IRCOM-SIC laboratory of Poitiers (France) jointly with the IETR in Rennes (France). The aim is to find an efficient transmission system, in terms of visual quality for the received image. Usual source coding schemes are inappropriate when errors occur during the transmission. Many errors correction systems exist but they significantly decrease the compression ratios for a low errors resistance. To solve this problem, a Vector Quantization (VQ) algorithm based on the Kohonen topological maps (SOFM: Self Organization Feature Map) is used. The image used for testing is shown in fig. 13.29. 


\subsubsection{Results}

Several acquisitions were made on 20th February 2003 at frequencies between 6 and $9 \mathrm{MHz}$. For this experiment, the signal bandwidth was limited to $3 \mathrm{kHz}$. The modulation is a QAM-16 and the symbol's duration is $0.4 \mathrm{~ms}$. The transmitted files duration varies between $4.9 \mathrm{~s}$ and $5.8 \mathrm{~s}$. They are sent continuously over $20 \mathrm{~s}$. The number of acquisition files is 72 . For many reasons, some of them are unusable: too weak signal due to bad propagation conditions, jammer or important noise pulse, etc. The number of useable files is 57 and table 13.I presents a list of the performances.

The results obtained at the first carrier frequency $(6.99 \mathrm{MHz})$ are rather good: the constellation points are clean (fig. 13.27) and the BER is small.

When using the second and third carrier frequencies $(7.97 \mathrm{MHz}$ and $8.91 \mathrm{MHz}$ ), the results are not flawless: the constellation points are not as clean as in the previous example (fig. 13.28) and the BER is larger.

The image in the fig. 13.30d contains twice as much errors than others images. However, the coding gain is $7.19 \mathrm{~dB}$ in relation to SPIHT (fig. 13.30c), $8.13 \mathrm{~dB}$ in relation to JPEG2000 (fig. 13.30b) coding and $18.14 \mathrm{~dB}$ in relation to JPEG (fig. 13.30a). For the same compression rate, the SOFM associated with QAM modulation fits very well for transmission through the ionospheric channel.

Table 13.I. Number of data files recorded at 3 frequencies and corresponding BER.

\begin{tabular}{|c|c|c|}
\hline Carrier frequency & BER interval & Number of files \\
\hline $\begin{array}{l}\text { 6.994 MHz } \\
\text { (27 files) }\end{array}$ & $\begin{array}{c}0 \leq \mathrm{BER} \leq 10^{-4} \\
10^{-4}<\mathrm{BER} \leq 10^{-3} \\
10^{-3}<\mathrm{BER} \leq 10^{-2} \\
\text { Unusable }\end{array}$ & $\begin{array}{c}21 \\
0 \\
0 \\
6\end{array}$ \\
\hline $\begin{array}{l}\text { 7.967 MHz } \\
\text { (22 files) }\end{array}$ & $\begin{array}{c}0 \leq \mathrm{BER} \leq 10^{-4} \\
10^{-4}<\mathrm{BER} \leq 10^{-3} \\
10^{-3}<\mathrm{BER} \leq 10^{-2} \\
\text { Unusable }\end{array}$ & $\begin{array}{c}2 \\
16 \\
3 \\
1\end{array}$ \\
\hline $\begin{array}{l}\text { 8.917 MHz } \\
\text { (8 files) }\end{array}$ & $\begin{array}{c}0 \leq \mathrm{BER} \leq 1.10^{-4} \\
10^{-4}<\mathrm{BER} \leq 10^{-3} \\
10^{-3}<\mathrm{BER} \leq 10^{-2} \\
\text { Unusable }\end{array}$ & $\begin{array}{l}0 \\
5 \\
3 \\
0\end{array}$ \\
\hline
\end{tabular}

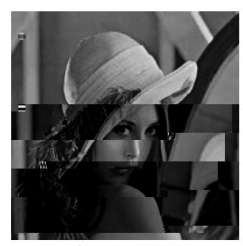

(a) JPEG

PSNR $=11.61 \mathrm{~dB}$

$\mathrm{BER}=\mathbf{1 . 9 6 \cdot 1 0 ^ { - 4 }}$

(14 errors)

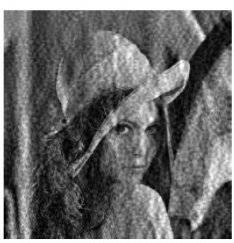

(b) JPEG2000

PSNR $=21.62 \mathrm{~dB}$

BER $=3.22 \cdot 10^{-4}$

(23 errors)

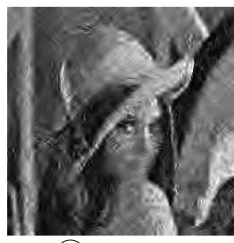

(C) SPIHT

PSNR $=22.56 \mathrm{~dB}$

BER $=3.5 \cdot 10^{-4}$

(25 errors)

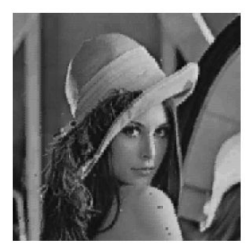

(d) SOFM

PSNR $=29.75 \mathrm{~dB}$

$\mathrm{BER}=\mathbf{7 . 0 \cdot 1 0 ^ { - 4 }}$

(50 errors)

Fig. 13.30a-d. Images received using different number of antennas. 


\subsection{ConClusions AND OUTLOOKS}

This paper presents a way to achieve a significant improvement of the data transfer rate in a HF radio link by the use of a heterogeneous array. The actual rate of the system reaches up to $20 \mathrm{kbits} / \mathrm{s}$ (in $6 \mathrm{kHz}$ bandwidth) whereas the current standard for scalar modems is $4.8 \mathrm{kbits} / \mathrm{s}$ in a $3 \mathrm{kHz}$ bandwidth. Actually, only one link has been tested with a relatively short range and limited number of paths. Moreover, no coding, interleaving or soft decision techniques were employed to reach these performances. The results obtained in terms of BER (about $10^{-3}$ ) are considered to be acceptable for HF modems. With a 4 circular antenna array, a QAM modulation and a robust source coding, the SOFM method fits very well for transmission through the ionospheric channel.

Future work will consider longer range links. In this context, the number of received signals will increase in relation to the number of ionospheric paths so that the demodulation processing will have to cope with more severe conditions of reception.

In addition, investigations will be performed to adjust the transmitted polarization in order to reduce the number of modes propagating through the ionosphere and this will reinforce the spatial filtering.

Another development will be the implementation of a blind algorithm for spatio-temporal equalization in the receiving system, replacing the LMS algorithm. The benefit will be to avoid the transmission of training sequences and, consequently, to increase the effective data transfer rate.

\section{REFERENCES}

Bisiaux, A. (2001): Définition, conception et réalisation d'un modem vectoriel large bande utilisable dans la gamme Hautes Fréquences, Ph.D. Thesis (Université de Rennes 1).

BisiauX, A. and L. BERTEL (2000): Antenna diversity as a means of improving HF transmissions, in Proceedings of the IEE «HF Radio Systems and Techniques», Guildford, U.K.

BuDdEn, K.G. (1985): The Magneto-Ionic Theory and its Applications to the Ionosphere (Cambridge University Press).

ERHEL, Y.M., L. BERTEL and F. MARIE (1998): A method of direction finding operating on an array of collocated antennas, in IEEE-AP International Symposium, Atlanta.

MARIE, F., Y.M. ERhEL, L. BERTEL and D. LEMUR (2000): Design of a HF compact direction finding system based on collocated antennas, in Proceedings of the IEE, HF Radio Systems and Techniques, Guilford, U.K.

Mengali, U. and A. D’Andrea (1997): Synchronization Techniques for Digital Receivers (Kluwer Academic/Plenum Publishers).

Souhard, B., C. Chatelier and C. Olivier (2003): On the robustness of joint source/channel coding for transmission through an ionospheric channel, in Proceedings of the 9th International Conference on HF Radio Systems and Techniques, June 2003, Bath, U.K.

WiDROw, B. and J.M. MCCOLL (1976): A comparison of adaptative algorithms based on the methods of steepest descent and random search, IEEE Trans. Ant. Prop., 24, 615-637. 